\title{
Um sistema de indicadores para comparação entre organizações: o caso das pequenas e médias empresas de construção civil
}

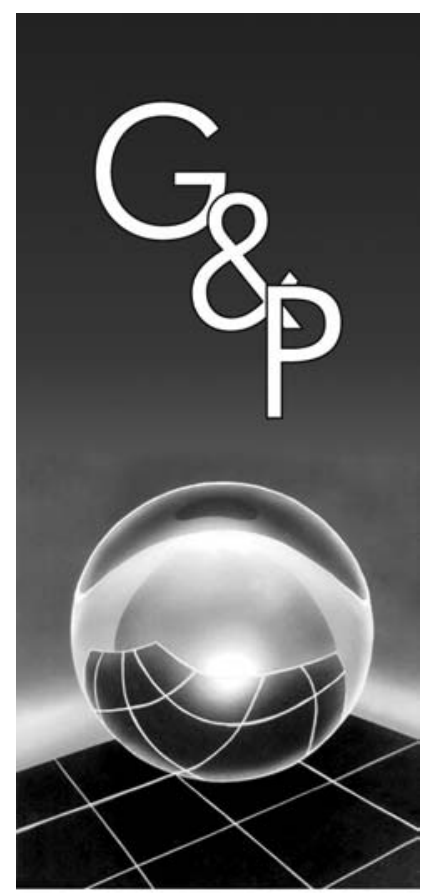

\author{
Luiz Carlos Brasil de Brito Mello \\ Sérgio Roberto Leusin de Amorim \\ Renata Albergaria de Mello Bandeira
}

Resumo

Este trabalho avalia o desempenho das médias e pequenas empresas (PME) de construção civil que utilizam critérios de medição e sistemas de indicadores em relação àquelas que não utilizam, confrontando os resultados obtidos nas dimensões: finanças, recursos humanos, mercado e produção. Para tanto, partiu-se do pressuposto de que a implantação de indicadores e critérios de medição em PMEs da construção civil traz benefícios. Desta forma, foram aplicados os indicadores selecionados em uma amostra de pequenas e médias empresas da construção civil, certificadas e não certificadas, situadas no município do Rio de Janeiro. A pesquisa realizada demonstrou que as empresas que utilizam critérios de medição apresentam melhores resultados.

Palavras-chave: Pequenas e médias empresas. Construção civil. Indicadores.

\section{Introdução}

O setor de construção civil se caracteriza pela concentração de microempresas: $58 \%$ das empresas de edificações concentram-se na faixa de microempresas (até 9 empregados), seguidas do grupo de pequenas empresas (entre 10 e 99 empregados) com 33\% (SENAI, 1999). Entretanto, as empresas de pequeno e médio porte são comprimidas entre as grandes empresas que dominam o mercado de alta renda e pelo setor informal que lhes rouba os consumidores de renda baixa e média. Desta forma, só resta às pequenas e médias empresas (PMEs) rever seus processos e produtos de maneira que possam continuar competindo. Algumas pequenas e médias empresas da construção civil entenderam que a implantação de um sistema de gestão da qualidade (SGQ) pode qualificá-las para um posicionamento mais eficaz para enfrentar os desafios existentes.

No entanto, para se implantar um Sistema de Gestão de Qualidade há não só um custo inicial alto como também o custo de manutenção do sistema. Tal fato, em muitos casos, é proibitivo para as pequenas e médias empresas. Problemas financeiros decorrentes de aspectos como juros altos, falta de acesso ao crédito e baixa produtividade já dificultam a continuidade destas empresas. Como, então, investir em sistemas que podem melhorar o seu desempenho, se inicialmente os custos aumentam? Desta forma, a motivação para esta pesquisa surgiu da curiosidade de se avaliar os resultados obtidos por PMEs da construção civil que utilizam SGQs, comparativamente àquelas que não os utilizam. Foi desenvolvido, então, um sistema de indicadores que estão relacionados às seguintes dimensões: finanças, recursos humanos, mercado e produção.

O escopo deste trabalho consiste em avaliar o desempenho das médias e pequenas empresas de construção civil que utilizam critérios de medição e sistemas de indicadores em relação àquelas que não utilizam, confrontando os resultados obtidos nas dimensões: finanças, recursos humanos, mercado e produção. Para tanto, partiu-se do pressuposto de que a implantação de indicadores e critérios de medição em PMEs da Construção Civil traz benefícios. As organizações tornam-se menos suscetíveis a imprevistos e, portanto, podem se organizar melhor, fazendo um planejamento mais adequado. Através de medições conseguem verificar quais os pontos deficientes, permitindo que sejam feitos planos para a melhoria contínua. Supõe-se, também, que as PMEs que não utilizam indicadores e critérios de medição apre- 
sentam resultados piores do que aquelas que tenham implantado tais instrumentos. Dentro deste contexto adotou-se a hipótese de que as empresas de pequeno e médio porte do ramo de construção civil, que utilizam indicadores e critérios de medição, apresentam melhores resultados do que aquelas que não os utilizam.

\section{Sistemas de medição}

Mears (1993) define um sistema de gestão da qualidade como um processo permanente e de longo prazo direcionado para a satisfação do cliente, que permite a melhoria contínua dos produtos e serviços gerados. $\mathrm{O}$ sistema de gestão da qualidade encara o processo de produzir bens ou serviços como um gerador potencial de vantagem competitiva organizacional. Tal visão permite que haja uma grande influência na definição das estratégias operacionais, que objetiva produtos e serviços sem erros, entregues no prazo e dentro dos custos, além da diminuição dos prazos de introdução de novos produtos, maior oferta de produtos e facilidade de mudanças de prazos. Com a utilização de sistemas de gestão da qualidade, consegue-se um perfeito entrosamento entre as estratégias competitivas e as de construção e fabricação.

No atual estágio da indústria da construção civil, é necessário que exista um aperfeiçoamento da capacidade de absorção das empresas perante as inovações tecnológicas. Para que tal fato ocorra, é importante que haja envolvimento nas inovações organizacionais e nos processos de aprendizagem visando a plena utilização de inovações como: tecnologias de informação e comunicação, valoração ambiental, construção enxuta, times multifuncionais e tantas outras. A adoção de sistemas de gestão da qualidade permite que as organizações estabeleçam tais procedimentos, organizem seus processos e iniciem o gerenciamento do conhecimento, além de estabelecer mecanismos de feedback que permitem a identificação de problemas e possíveis soluções.

Sistemas de gestão da qualidade são baseados na medição, correção e aprimoramento dos desvios dos diferentes processos organizacionais. Estes sistemas têm como objetivo o aprimoramento contínuo dos processos, produtos e serviços. Assim, os colaboradores das organizações devem estar capacitados em gestão de processos e devem enxergar a melhoria como um processo, de maneira que inovem através da eficácia de processos, produtos e serviços. O processo de melhoria contínua diferenciará uma organização de outra. Aquela que estiver mais bem aparelhada a corrigir suas deficiências de processos, competências e produtos/serviços se apresentará ao mercado em melhores condições para suplantar as concorrentes.

O conhecimento do nível de maturidade das empresas em relação ao estágio de melhoria permite que as PMEs da indústria da construção civil desenvolvam estratégias para que os diversos níveis organizacionais evoluam em sua capacitação. O conhecimento do estágio em que se encontram é o primeiro passo para a melhoria. Entende-se, então, que as organizações devem possuir sistemas de gestão da qualidade eficazes, que lhes permitam adquirir vantagens competitivas que as diferenciem no mercado. Atualmente, possuir um Sistema de gestão da qualidade é uma necessidade vital que fortalece os métodos gerenciais das empresas. $\mathrm{O}$ aperfeiçoamento constante deste sistema de gestão faz parte da inovação tecnológica. A existência de sistemas de gestão da qualidade nas empresas de construção civil deverá permitir que sejam traçadas estratégias mais eficazes. No entanto, a implantação destes sistemas implica em que as empresas possuam uma avaliação sistemática de seu desempenho. Através desta avaliação, será possível estabelecer metas, identificar problemas e aprimorar processos. Entretanto, para que isto seja possível, é necessário que sejam estabelecidos indicadores que possibilitem esta medição e permitam comparações. Desta forma, para que um sistema de gestão da qualidade obtenha êxito, é necessário que as organizações adotem sistemas de medição.

Observa-se que diversos programas e prêmios de qualidade e produtividade têm exigido das organizações um monitoramento e avaliação de seus sistemas de gestão da qualidade. No Brasil, a Fundação Prêmio Nacional da Qualidade através do Prêmio Nacional da Qualidade, incentiva a medição do desempenho, principalmente pelo critério de foco nos resultados. Assim, a organização avalia um conjunto de indicadores que, alinhados a estratégias, refletem os planos e as metas organizacionais, as necessidades e os interesses de todas as partes interessadas (FUNDAÇÃO PRÊMIO NACIONAL DA QUALIDADE, 2004). Na Indústria da Construção Civil, apenas o Escritório de Engenharia Joal Teitelbaum ganhou, em 2003, o Prêmio Nacional da Qualidade na categoria médias empresas.

Como afirma Bethlem (2004), "para que o desempenho empresarial seja gerenciado, é necessária a sua medição". Salienta-se, entretanto, que não basta existir um sistema de medição de desempenho para aumentar o desempenho organizacional. É essencial que este esteja direcionado às estratégias e processos do negócio. Autores como Lynch (1995) e Costa (2003) afirmam que, em muitas empresas, os indicadores não são selecionados de acordo com os objetivos estratégicos e fatores críticos. Desta forma, há dificuldades na implementação do sistema de medição.

Rodriguez (2002) ressalta que, ao se estabelecer uma metodologia de medição, esta deve ser considerada como uma maneira de ajustar ou transformar a organização, não apenas uma forma de medi-la. $\mathrm{O}$ autor propõe que os indicadores devem se basear em objetivos criados segundo a abordagem top-down. Os indicadores devem 
ter um número limitado por gerência, unidade de negócio ou processo de trabalho. Ainda, todos na organização devem ter um entendimento das várias inter-relações entre as diversas dimensões críticas. Além dos cuidados sugeridos, existem alguns problemas, segundo Rodriguez (2002) e Costa (2003), para a implantação de indicadores, tais como: i) estratégia mal definida; ii) existência de sistemas de medição pré-existentes; iii) problemas de poder; iv) comunicação ineficaz; v) priorização de outras iniciativas; vi) dificuldade de coleta de dados; e vii) falta de uniformização no cálculo dos indicadores.

Costa (2003) e Lantelme (1994) apontam que os indicadores ainda não são utilizados por um grande número de empresas do setor de construção. Lantelme (1994) acredita que esta baixa utilização se deve aos seguintes fatores: i) a dificuldade que as empresas têm para identificar quais os indicadores críticos em relação às estratégias e fatores críticos de sucesso; ii) tendência à acomodação, por parte das empresas, quando constatam que possuem valores melhores que os de referência do setor; iii) a utilização do sistema de medição como uma forma de controle e não como uma forma de comunicação dos objetivos, divisão de responsabilidades e obtenção de maior participação e; iv) visão de curto prazo da gerência que busca resultados imediatos, esquecendo que a implementação de um sistema de medição requer tempo até o aparecimento dos resultados.

As empresas de projeto e de construção que utilizam sistemas de medição costumam optar por indicadores mais simples, com dados fáceis de serem obtidos, sem considerar quais informações são realmente críticas para a mensuração da melhoria contínua (OLIVEIRA; FREITAS, 2000; LANTELME, 1994). Barros Neto (1999) ressalta, ainda, a dificuldade da implantação de indicadores devido ao aspecto único do projeto e das condições locais, além da extrema variabilidade das condições externas, típicas da construção civil. Todavia, fica claro que a concepção, implementação e utilização de indicadores pelas pequenas e médias empresas da construção civil é uma necessidade para que possam manter os esforços de melhoria contínua e aprimoramento. Se não houver meios de se medir os processos, verificar as diferenças entre o planejado e o realizado e nem quantificar os ganhos de produtividade e qualidade obtidos, torna-se difícil o incentivo para o uso de tais sistemas.

A utilização de indicadores nas empresas vem se modificando nas últimas décadas. Anteriormente, havia preferência por medidas em que o desempenho das organizações era avaliado em termos de produtividade física ou financeira (por exemplo: volume de produção, lucro, etc.) e a preocupação era a eficiência técnica. Esta preferência pode ser creditada ao fordismo e ao taylorismo, que eram as práticas gerenciais vigentes. Autores como Ghalayini et al. (1997) consideram que a utilização deste tipo de medidas traz conseqüências como: i) elevado custo; ii) rápida desatualização; e iii) quantificação difícil de itens como: redução de prazos de entrega, satisfação dos clientes e outros, em termos monetários.

Costa (2003) considera que o modelo tradicional de indicadores não permite que as empresas avaliem sua competitividade e melhoria contínua no atual ambiente de negócios, muito competitivo e mutante. Ademais, um outro fator que trouxe necessidade de mudança dos indicadores foi a introdução de novas tecnologias de gestão e produção como garantia da qualidade total, just in time, Manufatura Flexível e outras que, necessitando mensurar conceitos como flexibilidade, redução de estoques, parcerias com fornecedores e requisitos dos clientes, mostraram a importância da introdução de novos indicadores que avaliassem estas novas dimensões. Uma série de indicadores é utilizada para que se meça o grau de eficácia ou eficiência de um processo.

$\mathrm{O}$ acompanhamento dos indicadores deve ser feito no nível estratégico da organização, a partir dos indicadores de resultado. É recomendável que um conjunto pequeno e balanceado de indicadores seja acompanhado pelo nível mais alto da organização. Rodriguez (2002) sugere que é razoável ter até dez indicadores que, quando desdobrados, podem gerar vários outros, devendo estar sempre alinhados com os indicadores de resultado. Estes é que servirão para indicar os caminhos da organização.

$\mathrm{Na}$ literatura pesquisada, verifica-se a existência de importantes trabalhos relacionados a indicadores de desempenho, tais como Lima (2005), Costa (2003), Santana (1997), Moreira (1996), Souza (1996), Lantelme (1994), Formoso et al. (1994). Formoso et al. (1994) classificam a medição de desempenho conforme a finalidade do indicador utilizado, podendo-se subdividir em: medições para visibilidade, medições para controle e medições para melhoria. Os autores ressaltam que as empresas com melhores resultados no processo de implantação do sistema de indicadores foram aquelas que distribuíram a atividade de coleta de dados por vários setores, segundo o relacionamento destes com a informação a ser coletada.

Outra pesquisa relevante é a de Souza et al. (1995), na qual é apresentada uma lista de indicadores de desempenho que é organizada em: indicadores de qualidade globais e específicos, indicadores de produtividade globais e específicos. O sistema é completo e extenso, funcionando como uma listagem de indicadores que podem ser aplicados. Todavia, devem ser adequados à realidade de cada empresa.

Lantelme (1994) propõe um sistema para a construção civil que é organizado em indicadores de qualidade e produtividade relacionados a: projeto, suprimentos, assistência técnica, planejamento e vendas, produção, recursos humanos e administrativos. Quando o trabalho foi realizado, as normas ISO 9000:1994 estavam 
sendo implementadas, e sua visão era diferenciada da atual NBR ISO 9001:2000. Entretanto, o trabalho de Lantelme (1994) foi uma importante contribuição para o início da discussão da cultura da qualidade no setor. O sistema proposto apresenta um formato semelhante ao de Formoso et al. (1994) e Oliveira et al. (1995). Costa (2003) colaborou para a evolução do sistema proposto por meio do desenvolvimento de um trabalho onde são agrupadas diretrizes para concepção, implementação e uso de sistemas de indicadores de desempenho em empresas da construção civil. A autora descreve as etapas a serem realizadas para esta utilização e faz referência a um estudo realizado por Lantelme (1999), cujo objetivo era o entendimento das barreiras para a implementação de sistemas de medição de desempenho em empresas de construção civil.

Dentre os sistemas de indicadores utilizados na construção civil, deve ser destacado o Sistema de Acompanhamento da Qualidade e Produtividade na Construção (QUALIPRO, 1998), desenvolvido pelo Clube da Qualidade na Construção. Esse trabalho originou o grupo de pesquisas NITCON*, gerando diversos trabalhos importantes sobre o assunto, tais como: Amorim (1998), Santana (1997), Souza (1996) e Amorim (1995). O trabalho de Souza (1996) faz referências ao programa de banco de dados QUALIPRO. No desenvolvimento do trabalho do QUALIPRO (1998) foram criados quatro grupos de indicadores: produtividade dos recursos humanos (tempos produtivos, improdutivos e auxiliares); produtividade dos recursos materiais (perdas e desperdícios dos materiais); qualidade do produto (avaliação pós-entrega); e qualidade dos serviços (retrabalhos). Moreira (1996) apresenta uma série de considerações a respeito da importância da medição de desempenho. A autora conclui que um sistema bem definido de medição aumenta o desempenho por oferecer um referencial que serve de base para a tomada de melhores decisões, evitando-se decisões baseadas em falsas suposições ou em objetivos pessoais, o que prejudica a qualidade e produtividade.

Diversos modelos de sistemas de medição de desempenho têm sido propostos a partir da década de 90 (CROSS; LYNCH, 1988; SINK; TUTTLE, 1993; LYNCH; CROSS, 1995; KAPLAN; NORTON, 1997; NEELY et al., 1997; SCHIEMANN; LINGLE, 1999). Autores como Sink e Tuttle (1993), Neely et al. (1992), Kaplan e Norton (1997) e Schiemann e Lingle (1999) sugerem a integração dos sistemas de medição de desempenho aos sistemas de gestão das empresas, considerando a medição como o elemento essencial para monitoramento e controle das estratégias. Todavia, Oliveira et al. (1993) consideram que

\footnotetext{
*Grupo de Pesquisas do Programa de Pós-Graduação em Enge-
} nharia Civil da Universidade Federal Fluminense. “...o setor da construção civil (...) encontra-se carente de dados que possam fornecer ao pessoal envolvido na realização de determinado processo, as informações quanto ao desempenho atual de suas empresas e orientar estratégias para a melhoria do desempenho global e as atividades rotineiras da empresa." (OLIVEIRA et al., 1993).

Mesmo tendo ocorrido uma evolução do setor na utilização de sistemas de gestão da qualidade, o que possibilita que as informações sejam rastreadas, tal afirmação ainda permanece atual. Esta condição pode ser comprovada pela indisponibilidade de dados importantes para possibilitar uma avaliação mais aprofundada do desenvolvimento da indústria da construção civil nos últimos anos.

A adoção de sistemas de indicadores de desempenho auxilia no atendimento dos requisitos de medição e monitoramento de produtos e processos prescritos no item oito da ISO 9001:2000 e, conseqüentemente, na melhoria dos sistemas de gestão da qualidade. Logo, as empresas construtoras com sistema de gestão da qualidade implantado têm, geralmente, um controle maior em relação aos seus processos e produtos. Contudo, estes indicadores nem sempre são adequados às suas necessidades (AMORIM, 1998).

A importância da utilização de indicadores nas organizações é um consenso entre os diversos pesquisadores da área. Para o sucesso da implementação de um sistema de indicadores, é fundamental que se tenham as estratégias da empresa em mente bem como os objetivos a serem alcançados.

Kaplan e Norton (1997), ao descreverem a metodologia do Balanced Scorecard (BSC), criaram um marco nos sistemas de indicadores. O BSC consiste na medição do desempenho de empresas com base em indicadores financeiros e não financeiros. Trata-se de um sistema de avaliação de desempenho empresarial. Seu principal diferencial consiste no reconhecimento de que indicadores de caráter somente financeiros não são suficientes por mostrarem apenas os resultados dos investimentos e das atividades, não contemplando os impulsionadores de rentabilidade de longo prazo.

Acredita-se que a implantação de sistemas de gestão da qualidade nas empresas construtoras colabore para a melhoria de seus desempenhos organizacionais. No entanto, percebe-se uma carência de ferramentas que facilitem a mensuração do desempenho das organizações. Os sistemas de indicadores comumente desenvolvidos nos trabalhos relativos à construção civil medem, geralmente, aspectos operacionais. O objetivo destes indicadores é avaliar o desempenho das obras realizadas pela empresa. A pesquisa desenvolvida por Fonseca (2006) preocupou-se em possibilitar a comparação dos dados da empresa com outras organizações e, 
também, com dados setoriais. Desta forma, é permitida a comparação do desempenho da organização com os valores de referência. Este conjunto de indicadores será utilizado no presente trabalho para comparar o desempenho das organizações certificadas em relação às que não são certificadas. Em seguida, é feita uma descrição do sistema de indicadores a ser utilizado na comparação entre as empresas.

\section{Desenvolvimento da proposta de indicadores}

Inicialmente, para a proposição preliminar do sistema, foram discutidas algumas características que um sistema de indicadores deve conter, com base em relatos de outros autores e experiências anteriores de empresas que colaboraram no desenvolvimento de tal sistema. Além disso, foram considerados os processos básicos utilizados que necessitam de controle. As condições consideradas para determinar a viabilidade do conjunto de indicadores foram: a produção de informações a baixo custo, o suporte na tomada de decisões, o fácil entendimento, a aceitação e o uso rotineiro por todos na empresa. Foram consideradas, também, as sugestões de Hronec (1994), que identifica três níveis de desempenho na empresa para o desenvolvimento dos indicadores: i) Humano: as pessoas que executam as atividades; ii) Processo: definido como uma série de atividades que consomem recursos e fornecem produtos aos clientes da empresa (internos e externos); e iii) Organização: compreende, simultaneamente, os níveis de desempenho das pessoas e do processo.

O sistema de indicadores elaborado por Fonseca (2006) tem como foco os indicadores organizacionais, que proporcionam um acompanhamento da organização, auxiliando na elaboração de novas estratégias. As idéias de Kaplan e Norton (1997) também serviram como base para considerações referentes ao aperfeiçoamento do sistema de indicadores. Os princípios do BSC sugerem que os objetivos e medidas derivam da visão e da estratégia da empresa, focalizando o desempenho organizacional sob quatro perspectivas: i) financeira; ii) clientes; iii) processos internos da empresa; e iv) aprendizado e crescimento. As medidas e os objetivos financeiros definem o desempenho financeiro esperado da estratégia, servindo de meta principal para os objetivos e medidas de todas as outras perspectivas; as perspectivas dos clientes devem alinhar suas medidas de resultados relacionadas aos clientes (satisfação, fidelidade, captação) com segmentos específicos de clientes e mercados. Em relação à perspectiva dos processos internos da empresa, Kaplan e Norton (1997) sugerem que seja definida uma cadeia de valor dos processos internos, que deve ter início com o processo de inovação, passando pelos processos de operações e finalizando com o serviço pós-venda. A perspectiva de aprendizado e crescimento desenvolve objetivos e medidas para orientar o aprendizado e o crescimento organizacional.

Considerando-se os requisitos mencionados, optou-se pela utilização de quatro categorias de indicadores considerados fundamentais para a aferição do desempenho das empresas: financeiro, recursos humanos, mercado e produção. Os indicadores foram organizados desta forma pela crença de que são itens vitais para a avaliação do desempenho e perenização de uma empresa.

Fonseca (2006) considera que, resguardadas as características encontradas na indústria da construção civil, existe uma similaridade entre as quatro perspectivas sugeridas por Kaplan e Norton (1997) e as quatro categorias de indicadores propostas. Da mesma maneira que Kaplan e Norton (1997) ressaltam a importância da perspectiva financeira para a análise do desempenho organizacional, inclui-se no sistema um conjunto de indicadores de aspecto financeiro. Os objetivos e medidas relacionados à perspectiva dos processos internos da empresa podem ser relacionados à categoria de produção. A perspectiva dos clientes assemelha-se ao objetivo da apuração de indicadores da categoria de mercado e também de produção. A categoria recursos humanos pode ser vinculada com a perspectiva de aprendizado e crescimento, pois esta é desenvolvida por Kaplan e Norton (1997) em três categorias, entre elas capacidade dos funcionários. Deve ser ressaltado, entretanto, que estas considerações entre as perspectivas listadas no Balanced Scorecard e as categorias de indicadores de desempenho do sistema apresentado para as empresas é apenas ilustrativa. Os indicadores descritos em cada categoria possuem características próprias, voltadas para o perfil das empresas construtoras.

Definidas as categorias de indicadores a serem apuradas, iniciou-se a etapa de detalhamento do sistema, definindo os indicadores relevantes de cada conjunto, com seus respectivos objetivos. Os indicadores de desempenho foram criados e selecionados de acordo com a relevância, representatividade, viabilidade e facilidade de coleta.

Em relação aos aspectos financeiros, considerou-se importante a utilização de indicadores como rentabilidade e faturamento, além da correlação destes com a produtividade dos funcionários. Assim, existe a possibilidade de comparação entre empresas com níveis de investimento distintos, visto que somente o indicador de faturamento bruto de uma empresa não representa necessariamente um melhor desempenho ou melhor utilização de um sistema de gestão.

Os indicadores relacionados aos recursos humanos são fundamentais para a verificação da evolução do sistema de gestão de uma empresa e podem ser mensurados através de dados relativos a índices de acidentes, índices de rota- 
tividade e qualificação dos funcionários e pela existência de atitudes que demonstrem a preocupação com a responsabilidade social por parte da empresa. Lantelme (1994) ressalta que a implantação de um sistema de gestão da qualidade requer uma atenção especial para a questão dos recursos humanos. Segundo a autora,

\begin{abstract}
“...a qualidade em seu sentido amplo é entendida como a satisfação de todos os clientes, dentre eles os empregados. Assim, as condições de segurança e higiene do trabalho, treinamento e motivação não podem ser desconsideradas na busca de melhoria." (LANTELME, 1994).
\end{abstract}

Outro grupo de indicadores imprescindíveis para a avaliação do desempenho de uma empresa são os relacionados ao mercado, pois mensuram a capacidade de sobrevivência e crescimento organizacional. Para tanto, devem ser coletados dados como percentual de mercado, evolução de mercado e velocidade de vendas.

$\mathrm{Na}$ categoria de indicadores relacionados à produção, adotaram-se medidas referentes à eficiência do planejamento produtivo e índices de não conformidade, que são fatores importantes para o feedback de informações que dão suporte ao planejamento dos processos da empresa.

Em conjunto com a identificação dos indicadores de maior relevância para as empresas, considerou-se também a busca por dados equivalentes na indústria da construção civil, de forma a possibilitar a avaliação comparativa entre as construtoras e o restante do setor.

$\mathrm{Na}$ categoria de indicadores financeiros, para se avaliar a riqueza criada por empregado, utiliza-se o lucro bruto da empresa, denominado de valor agregado, relacionando o volume de entradas e saídas de capital da empresa, com o objetivo de corrigir possíveis distorções que pudessem ser geradas. Considerou-se que a apuração do valor de vendas pode sofrer interferências de fatores externos que poderiam gerar desvios na avaliação da participação dos funcionários na geração de receitas para a empresa. Além disso, é fundamental ressaltar que os números devem incluir a mão-de-obra terceirizada para que se obtenha um indicador representativo da realidade das obras.

Buscaram-se, ainda, maneiras mais coerentes para avaliar o endividamento e a lucratividade da empresa. No quesito lucratividade, adequou-se o indicador utilizado ao conceito de valor agregado, ao invés de lucro total da obra. Utilizou-se a fórmula para cálculo de endividamento que relaciona o passivo circulante e o exigível a longo prazo com o ativo total da empresa. O passivo circulante são as obrigações que normalmente são pagas dentro de um ano (curto prazo), enquanto o exigível a longo prazo relaciona-se às dívidas da organização que serão liquidadas com prazo superior a um ano. Em relação aos empregados, convencionou-se trabalhar com o número médio de funcionários por mês, incluindo-se os terceirizados. $\mathrm{O}$ número a ser inserido na fórmula seria o somatório de empregados próprios com os terceirizados, dividido pelos 12 meses do ano.

Os indicadores de recursos humanos incluem o índice de treinamento, em que é necessário apurar o total de horas dedicadas ao treinamento da empresa, incluindo-se funcionários da administração central, além da mãode-obra direta que opera nos canteiros de obra. Para a apropriação do número de horas trabalhadas, basta apurar o número de funcionários e multiplicar pelo número de semanas do período estudado, multiplicando por 44 horas semanais de trabalho.

Em relação aos indicadores de mercado, utiliza-se o índice de participação no mercado. O cálculo pode ser feito dividindo-se o faturamento da empresa pelo mercado total, obtido através de fontes como a ADEMI (2005) e o IBGE (2003). O indicador de velocidade de vendas se assemelha à apuração da rotatividade de um estoque.

Os indicadores de produção são compatíveis aos indicadores gerais existentes, possibilitando a realização de comparações entre os dados das empresas e os do setor. O cálculo do índice de qualidade do produto deve ser feito como o número de atendimentos das reclamações (considerou-se 90 dias após a entrega) em relação à área total e ao número de unidades vendidas. Divide-se o número de reclamações por um fator de unidades $/ \mathrm{m}^{2}$, de forma a contemplar as seguintes situações: comparando-se uma mesma metragem quadrada total produzida, mas com um número de unidades diferentes (ou seja, quanto maior o número de unidades, menor o tamanho de cada uma delas), a probabilidade de surgir reclamações é diferente. Com a fórmula apresentada, caso haja um mesmo número de reclamações nas duas situações, a que apresenta maior número de unidades terá um índice de qualidade do produto melhor, e vice-versa. Ressalta-se que, quanto menor o valor absoluto do indicador, melhor é o resultado deste, pois o objetivo é obter um número de reclamações o mais próximo de zero.

O índice de satisfação ao cliente considera a divisão entre o número de reclamações dos clientes (90 dias após a entrega) pelo número de unidades entregues. Considerou-se o número absoluto de reclamações como a melhor maneira de avaliar a satisfação do cliente, pois um número maior de reclamações, mesmo que de baixo custo de reparação, representa um impacto maior no atendimento das necessidades do cliente e na imagem da empresa.

$\mathrm{O}$ indicador de eficiência do planejamento produtivo é de grande utilidade para as empresas. Optou-se por instituir um indicador geral de produtividade da mão-de-obra, que relaciona o número de homens-hora utilizado em um determinado período com a metragem quadrada produzida no mesmo espaço de tempo.

Considerou-se que os demais indicadores de mercado propostos se adequiam melhor ao grupo de indicadores de produção. Realizaram-se modificações nos indicadores 
de produção com o intuito de torná-los compatíveis aos indicadores gerais existentes, possibilitando a realização de comparações entre os dados das empresas e os do setor.

Diante das considerações apresentadas, elaborou-se uma versão mais aprimorada para o sistema, com proposições de fórmulas para a obtenção dos indicadores de desempenho. Esta versão final é apresentada no Quadro 1.

Acredita-se que o sistema de indicadores a ser utilizado permite o estabelecimento de um cenário da indústria da construção civil, baseado em números oriundos de fontes confiáveis, evitando a elaboração de paradigmas fundamentados em intuições e experiências pessoais. A utilização de um sistema de indicadores de desempenho, focado na medição de aspectos estratégicos da empresa, permite a visualização da real situação da organização e a elaboração de planos de correção que forem necessários. A gestão empresarial baseada em indicadores pode ser considerada uma inovação organizacional que contribui para a melhoria contínua nas empresas e para a evolução do setor.

O sistema de indicadores de desempenho, apresentado no Quadro 1, serve para apuração de um volume representativo de dados junto às empresas. A posterior realização de análises estatísticas destes dados permite avaliações comparativas atendendo aos objetivos deste trabalho. O desenvolvimento dessa análise busca avaliar os motivos que levam ao melhor desempenho de uma empresa em relação às demais. Em seguida, é discutido o desenvolvimento da pesquisa.

\section{Desenvolvimento da pesquisa}

A unidade de análise são as pequenas e médias empresas de construção civil, certificadas e não certificadas, situadas no município do Rio de Janeiro. O objeto de análise é a comparação do desempenho de umas em relação às outras, utilizando-se o sistema de indicadores de desempenho proposto. Através de comparações entre os indicadores classificados nas dimensões finanças, recursos humanos, mercado e produção (Quadro 1), é possível, por comparações estatísticas, verificar o desempenho das PMEs certificadas em relação às não certificadas.

O delineamento utilizado na pesquisa foi: Revisão bibliográfica, Pré-teste, Estudos de caso e Interpretação dos resultados, com análises intra e intercasos. A definição da metodologia considerou aspectos peculiares da investigação e da análise de outras opções existentes. $\mathrm{O}$ modelo proposto levou em consideração aspectos práticos de seleção e tamanho de amostras. Para esta seleção, que será demonstrada adiante, foi utilizado o software estatístico Minitab-versão 13.1.

Nesta etapa, foram realizadas as validades de face e conteúdo (LITWIN, 1995). Foi realizado o pré-teste por meio da aplicação prévia dos indicadores (Quadro 1) a um grupo com as mesmas características da população a ser incluída na pesquisa. $\mathrm{O}$ sistema de indicadores foi validado em duas etapas. A primeira etapa foi a discussão dos indicadores propostos com três empresários do setor com formação acadêmica e vivência junto a pequenas e médias empresas do ramo imobiliário da indústria da

Quadro 1. Indicadores para comparação entre as empresas. (MELLO et al., 2008).

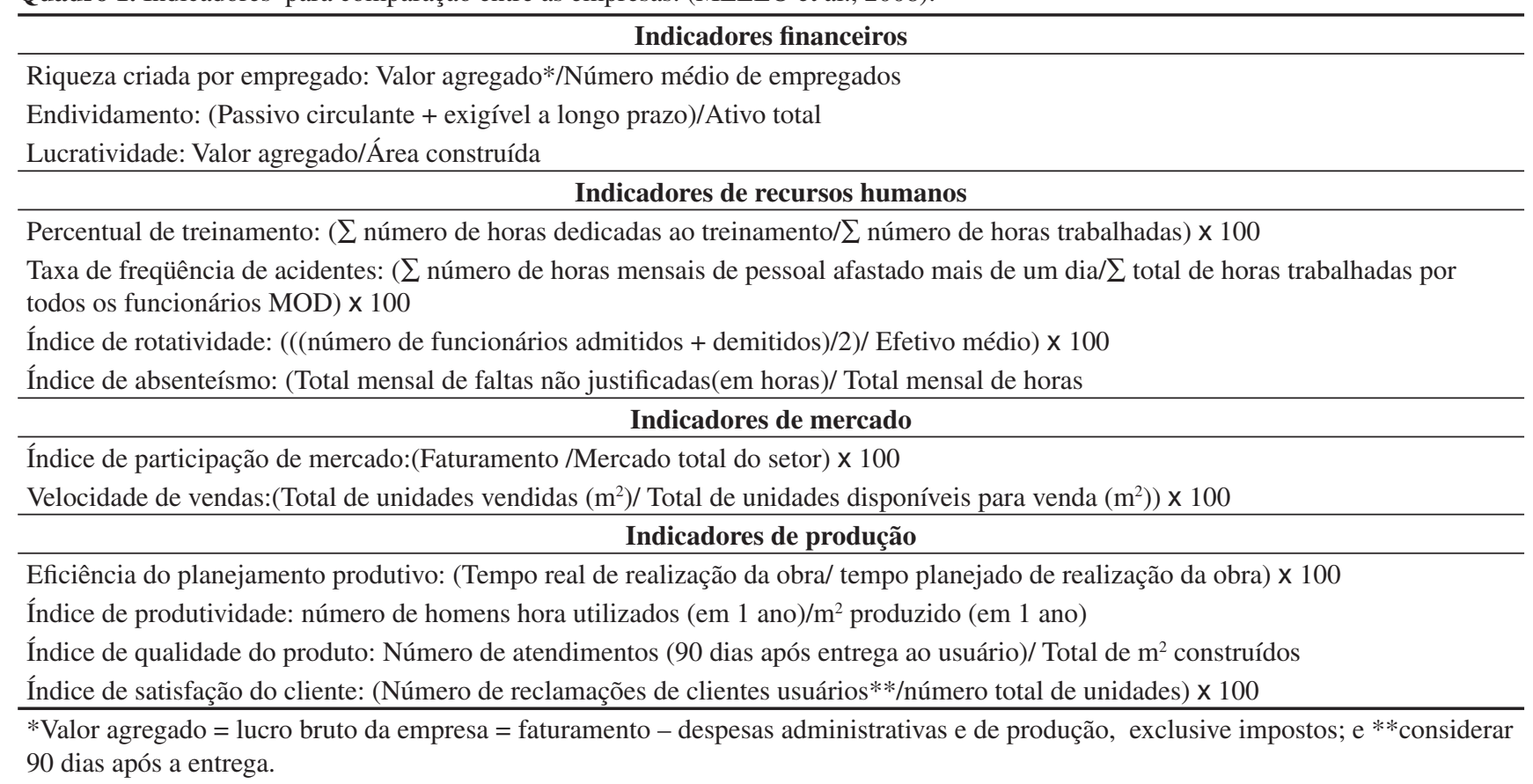


construção civil. A segunda fase abrangeu a aplicação dos indicadores em três empresas do setor escolhidas aleatoriamente (uma empresa com certificação A e duas empresas não certificadas). Portanto, o estudo de casos foi validado através dos fatores prescritos por Yin (2001): validade do construto (definido exatamente o que se quer estudar e descrever), validade interna (testada a coerência entre o que se quer estudar e os resultados obtidos), validade externa (estabelecido o domínio sobre o qual as descobertas possam ser generalizadas), e confiabilidade (verificada a possibilidade do estudo ser repetido). Foram, também, tomadas as devidas providências para que se evitasse a inconsistência dos dados.

\subsection{Descrição do método}

Inicialmente, foram definidos os grupos de empresas que serviriam de base para o estudo. Da listagem de empresas de serviços e obras qualificadas como A do PBQP-h, foram retiradas aquelas situadas no município do Rio de Janeiro (PBQP-h, abril 2006). Em seguida, foi feita a interseção entre a lista destas empresas e o Cadastro Industrial do Estado do Rio de Janeiro 20032004 (FIRJAN, 2003) para a obtenção da lista final de empresas atendendo aos dois critérios de seleção: i) empresas classificadas no PBQP-h com avaliação A, situadas no município do Rio de Janeiro; e ii) classificadas como pequenas ou médias empresas. Em abril/2006, sete empresas enquadraram-se nos critérios de seleção, constituindo o universo deste grupo.

Segundo o Cadastro Industrial do Rio de Janeiro 2003-2004 (FIRJAN, 2003), existem setenta e duas organizações de pequeno e médio porte no setor de construção civil, situadas no município do Rio de Janeiro, que não possuem sistemas de certificação e não estão incluídas na listagem de empresas de serviços e obras qualificadas (PBQP-h, 2006). O tamanho da amostra para este segundo grupo, determinado através do software estatístico Minitab-versão 13.1, foi de dezessete empresas, que foram selecionadas aleatoriamente entre as setenta e duas empresas existentes. Como se trata de dois grupos em que se trabalhou com as médias, utilizou-se o 2-sample t test ${ }^{*}$, com índice de confiabilidade (alfa) igual a 0,05 , sigma de 1 e uma potência de teste de 0,8 .

Após as modificações do quadro de indicadores, conforme os comentários recebidos na fase de pré-teste, iniciou-se a pesquisa junto aos dois grupos. Em seguida, foram feitos contatos com as empresas selecionadas objetivando sua adesão à pesquisa para apresentação da pesquisa e proposição de aplicação do sistema de indicadores. O trabalho iniciou-se em agosto de 2006, estendendo-se até novembro de 2006 com a realização da análise dos dados. Para os casos em que a análise dos

*2- sample $t$ é um teste utilizado para determinar se as médias de duas populações são iguais. dados primários apresentou discrepância, realizou-se nova entrevista para eliminá-la. Tais entrevistas foram conduzidas em dezembro de 2006.

Foi executada a triangulação de dados e de métodos por intermédio de levantamentos estruturados, entrevistas e observação do pesquisador, determinando-se a validade do construto. A validade externa da pesquisa foi evidenciada através da: i) escolha das empresas incluídas (pequenas e médias empresas de construção civil, certificadas e não certificadas, situadas no município do Rio de Janeiro); ii) escolha dos entrevistados; e iii) realização do estudo piloto em três empresas.

Como procedimento de análise dos indicadores, foram calculadas as médias para cada indicador em relação aos dois grupos amostrais (empresas certificadas e não certificadas). Verificou-se, então, a normalidade e, caso não fosse normal, utilizou-se teste não paramétrico Mann-Whitney**, o que permitiu comparar dois grupos independentes de dados. Além disto, foi utilizado o teste de Equal Variances ${ }^{* * *}$ para comparar as médias dos dois grupos.

\subsection{Informações gerais sobre as empresas (estudos de caso)}

As empresas não certificadas apresentam faturamento anual entre quatrocentos mil reais e três milhões novecentos e trinta e cinco mil reais. O número de empregados próprios deste grupo amostral varia de vinte a cem empregados, enquanto que o número de empregados terceirizados é de setenta funcionários na menor, e de até trezentos e cinqüenta empregados na maior organização. Estas empresas constroem em média mil metros quadrados/ano, a menor, e até trinta e cinco mil metros quadrados/ano, a maior. Esta amostra é constituída por $57 \%$ de empresas de pequeno porte e $43 \%$ de médio porte.

As empresas certificadas apresentaram um faturamento anual variando entre um milhão de reais e dois milhões e seiscentos mil reais. O número de funcionários próprios variou entre vinte e cem, enquanto que o número de funcionários terceirizados fica entre cento e setenta e duzentos e oitenta, na menor e na maior organização, respectivamente. As empresas certificadas construíram de dois mil e quinhentos metros quadrados anuais, a menor, até cento e dez mil metros quadrados anuais, a maior. Cinqüenta por cento das empresas certificadas, constituintes da amostra, são de médio porte.

A seguir, é descrita a análise estatística. Apresenta-se também a análise das principais observações feitas segundo os critérios gerais enunciados na metodologia.

\footnotetext{
**Mann-Whitney é um teste não paramétrico utilizado para comparar dois grupos independentes de dados.

***Teste de Equal Variances é um teste estatístico utilizado para comparar médias de dois grupos independentes.
} 


\section{Análise da pesquisa}

Os resultados demonstrados na Tabela 1 indicam que o desconhecimento dos dirigentes, a falta de conscientização do grupo e a falta de uma metodologia de aplicação são os maiores obstáculos para a aplicação de sistemas de indicadores nas empresas não certificadas, tendo recebido graus de dificuldade média para elevada. Ressalta-se que o grau 1 representa pouca dificuldade e o grau 5 representa muita dificuldade. Além disto, salienta-se que $85 \%$ das respostas das PMEs não certificadas vêm da Alta Direção. Alguns dirigentes também apresentaram como motivos de muita dificuldade o nível de educação e de

qualificação do grupo envolvido e o fato das empresas não possuírem recursos para ter profissionais habilitados na implantação dos critérios.

A visão das PMEs certificadas é diferente, pois as razões apresentadas no questionário foram pontuadas com graus de pouca dificuldade em sua grande maioria. Nas entrevistas, foram mencionados fatores de dificuldades como: a existência da grande rotatividade de pessoal no setor e a inexistência de dados confiáveis do setor para permitir comparações. Uma possível explicação para esta diferença de percepções é que as PMEs certificadas possuem, em sua estrutura organizacional, os aspectos tecnológicos para a melhoria da competitividade, citados por Ribault et al. (1995) e, assim, são melhor habilitadas para as mudanças do ambiente de negócios.

Observando as Figuras 1 e 2, verifica-se a similaridade de razões para a implantação de critérios de medição nas PMEs da construção civil. As empresas certificadas apresentaram, ainda, outros motivos como: exigência dos clientes e adequação à concorrência. $\mathrm{O}$ fato de terem introduzido a certificação antes das demais reafirma a percepção de que estas empresas estão mais sincronizadas ao ambiente externo, antecipando-se às mudanças e tomando as providências necessárias.

Utilizando o software estatístico Minitab versão 13.1 foram analisadas as respostas recebidas em relação aos indicadores apresentados no Quadro 1. Procurou-se validar ou não a hipótese considerada de que as PMEs da construção civil certificadas devem apresentar melhores resultados do que aquelas não certificadas. Para tanto,

Tabela 1. Dificuldades para implantar sistemas de indicadores nas PMEs da construção civil. (MELLO et al., 2008).

\begin{tabular}{lcc}
\hline \multicolumn{1}{c}{ Item } & $\begin{array}{c}\text { Não } \\
\text { certificadas }\end{array}$ & Certificadas \\
\hline Desconhecimento dos dirigentes & 4 & 1,8 \\
Falta de conscientização do grupo & 3,8 & 2 \\
Falta de metodologia de aplicação & 3,6 & 1,8 \\
Foi tentado e não deu resultado & 2 & 1 \\
Outros & - & - \\
\hline
\end{tabular}

empregou-se o teste 2-sample-t, por meio das médias calculadas e do cálculo do p-valor (probabilidade de que uma variável assuma um valor maior ou igual que o valor estritamente por acaso). A Tabela 2 apresenta as médias encontradas para os indicadores das duas amostras, bem como os $p$-valores calculados. Para todas as comparações o valor alfa adotado foi igual a 0,05 .

Tendo como ponto de partida o tratamento estatístico dos dados, foi elaborada a Tabela 3, que demonstra a situação das PMEs certificadas e não certificadas em relação aos diversos indicadores.

A partir da análise da Tabela 3, verifica-se que as PMEs certificadas apresentam resultados melhores em seis destes indicadores (riqueza, lucratividade, treinamento, taxa de acidentes, eficiência de planejamento e produtividade), quatro apresentam resultados semelhantes (endividamento, rotatividade, absenteísmo e participação no mercado) e três não permitem comparações, já que as

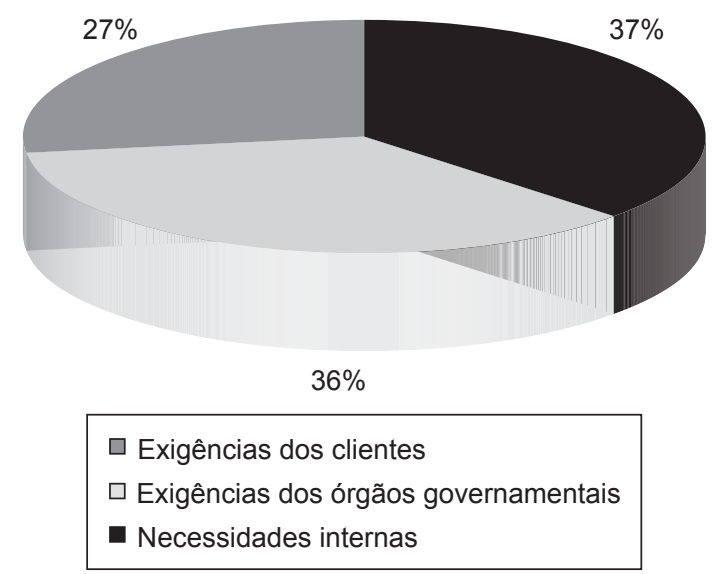

Figura 1. Principais razões para empresas não certificadas planejarem implantar SGQs.

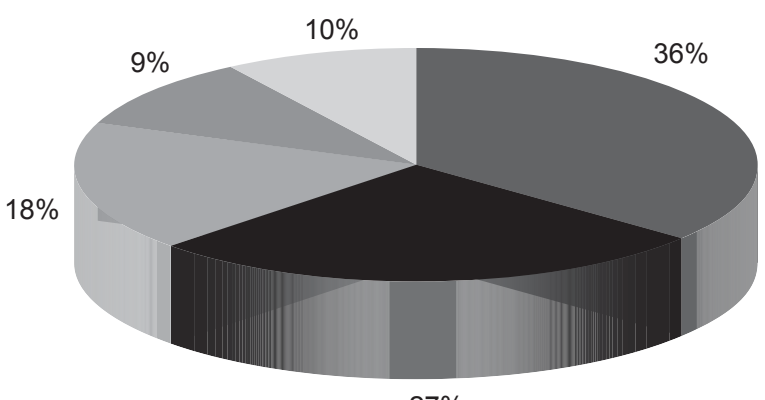

$27 \%$

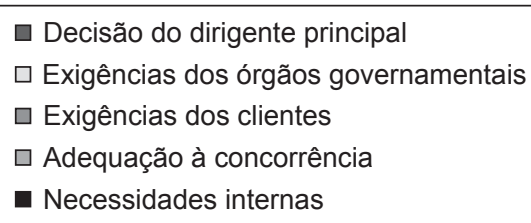

Figura 2. Principais razões para empresas certificadas terem implantado SGQs. 
Tabela 2. Médias dos indicadores para PMEs certificadas e PMEs não certificadas. (MELLO et al., 2008).

\begin{tabular}{llcl}
\hline Certificadas & \multicolumn{1}{c}{ Indicador } & $\begin{array}{c}\text { Não } \\
\text { certificadas }\end{array}$ & P valor \\
\hline 20.000 & Riqueza & 4942 & 0,000 \\
61,5 & Endividamento & 39,9 & 0,177 \\
275 & Lucratividade & 152 & 0,024 \\
1,03 & Treinamento & 0,083 & 0,000 \\
0,065 & Taxas de acidentes & 0,707 & 0,007 \\
51,2 & Rotatividade & 45,2 & 0,547 \\
1,83 & Absenteísmo & 7,10 & 0,055 \\
0,10 & Participação & 0,17 & 0,193 \\
& no mercado & & \\
64,63 & Velocidade de & ND & Não calculado \\
& vendas & & \\
100,17 & Eficiência no & 124,8 & 0,008 \\
& planejamento & & \\
30,6 & Produtividade & 52,8 & 0,033 \\
0,0055 & Qualidade & ND & Não calculado \\
93,92 & Satisfação & ND & Não calculado \\
\hline
\end{tabular}

Tabela 3. Comparação dos indicadores para PMEs certificadas e PMEs não certificadas. (MELLO et al., 2008).

\begin{tabular}{lll}
\hline Certificadas & \multicolumn{1}{c}{ Indicador } & Não certificadas \\
\hline Melhor & Riqueza & Pior \\
Semelhante & Endividamento & Semelhante \\
Melhor & Lucratividade & Pior \\
Melhor & Treinamento & Pior \\
Melhor & Taxas de acidentes & Pior \\
Semelhante & Rotatividade & Semelhante \\
Semelhante & Absenteísmo & Semelhante \\
Semelhante & Participação no mercado & Semelhante \\
S/C* & Velocidade de vendas & S/C* \\
Melhor & Eficiência no planejamento & Pior \\
Melhor & Produtividade & Pior \\
S/C* & Qualidade & S/C* \\
S/C* & Satisfação & S/C* \\
\hline
\end{tabular}

*Exigüidade de dados não permite comparações.

não certificadas não medem velocidade de vendas, qualidade e satisfação do cliente.

As causas apontadas para a não implantação de indicadores de medição foram: baixo nível educacional, falta de qualificação profissional e de recursos disponíveis para a implantação. Os problemas listados pelas PMEs não certificadas são validados por Grilo et al. (2003). Os autores declaram que os fatores mais resistentes à implementação de inovações na construção civil são: a escassez dos recursos humanos e materiais, altos custos diretos e indiretos incorridos no treinamento de colaboradores e líderes.

Deve ser ressaltado que as PMEs não certificadas não medem indicadores importantes sobre a qualidade do produto, rotação do estoque e percepção do cliente. Estes indicadores são medidos pelas PMEs certificadas. As PMEs não certificadas não têm dados que são utilizados em três indicadores. É importante destacar que, se os dirigentes não medem, não gerenciam. Salienta-se que o indicador Participação do Mercado deve ser relativizado, uma vez que os valores apresentados pelas empresas participantes da pesquisa, certificadas e não certificadas, não são consistentes e podem ter deturpado os resultados.

Outro ponto relevante destacado nas entrevistas com dirigentes das empresas não certificadas é que todos, inicialmente, dizem aplicar critérios de medição. No entanto, no decorrer da entrevista, quando solicitadas evidências objetivas, observa-se que não as possuem.

\section{Recomendações}

Os problemas encontrados nas análises feitas anteriormente em relação à não utilização de indicadores serão agrupados por similaridade, e serão propostas soluções. Para cada uma das recomendações toma-se o cuidado de procurar suporte na teoria identificada na revisão bibliográfica. Os principais problemas encontrados foram: i) PMEs não certificadas desconhecem critérios de medição e indicadores, não havendo direcionamento para a melhoria contínua; ii) as PMEs não certificadas não estão conscientes da necessidade de conhecer e aplicar critérios de medição e indicadores; iii) as PMEs não certificadas desconhecem a metodologia de aplicação de critérios de medição; iv) a produtividade das PMEs não certificadas é pior do que a das PMEs certificadas. O mesmo ocorre com a taxa de acidentes; e v) as PMEs não certificadas não analisam dados de qualidade e mercado, tais como: qualidade, velocidade de vendas e satisfação do cliente.

As soluções para os problemas relatados passam por uma interação maior entre universidades, sindicatos patronais (Sinduscons, ADEMI e outros) e organismos de apoio à pequena e média empresa como o SEBRAE para que sejam organizados grupos com a finalidade de discutir soluções objetivando a modernização das PMEs. Sugerem-se encontros com os empresários das PMEs em que os casos de sucesso de aplicação de indicadores e critérios de medição na construção civil sejam discutidos. Visitas técnicas, patrocinadas pelos organismos citados, devem ser feitas em empresas de outros ramos que sejam world class, para que os empresários sejam expostos às melhores práticas.

Devem ser criadas sinergias organizacionais que objetivem a criação de redes locais de PMEs da construção civil. Ao se integrarem em redes, as PMEs deixarão de atuar como pequenas empresas individuais, passando a constituir sistemas integrados de empresas. Com esta forma de atuação, as PMEs podem superar os problemas 
típicos de seu tamanho tais como: conhecimento especializado, inteligência empresarial, inovação, etc. No entanto, ao optarem pela formação de redes de cooperação, as PMEs serão obrigadas a enfrentar novas necessidades de capacitação e informação. Tais necessidades poderão ser supridas adotando-se as seguintes soluções: i) programas de capacitação em escolas técnicas e universidades; ii) programas de geração e difusão de novos conhecimentos, além de incorporar inovações técnicas e organizacionais. Desta forma, serão criadas capacitações para selecionar, adquirir, adaptar e assimilar novas soluções para os problemas decorrentes de desconhecimento e da não utilização de indicadores; iii) programas de capacitação para a utilização de recursos de inteligência organizacional e empresarial destinados à solução dos problemas apontados; e iv) facilitar o acesso e o intercâmbio de informações e o conhecimento de novos procedimentos, softwares e inovações técnicas e organizacionais necessárias para a solução dos problemas verificados pela pesquisa.

O estabelecimento das PMEs em redes permitirá o intercâmbio de idéias, o compartilhamento de informações, o conhecimento de tecnologias e práticas de organização. Várias estratégias e mecanismos são utilizados para promover a difusão de informações e conhecimento. Como exemplos podem ser citados: contatos interpessoais, cursos práticos, visitas a empresas, acordos para desenvolvimento, transferência de inteligência organizacional etc. Há vários exemplos de sucesso na literatura. Um deles é o programa belga conhecido como PLATO (ALBAGLI, 2001), em que empresários de pequenas e médias empresas reúnem-se em pequenos grupos para trocar informações em matéria de gestão e consultar administradores de grandes empresas sobre problemas específicos de gestão.

Também deverão ser criadas bases de dados estatísticos sobre as empresas, permitindo, então, que seja feito o benchmark entre elas. O benchmark deve ser utilizado para se inteirar do que outras organizações estão fazendo com bons resultados e procurar adaptar para as PMEs.

As associações empresariais (Sinduscons) podem trabalhar em conjunto com o SEBRAE e objetivar: i) a sensibilização e mobilização de segmentos sociais relevantes para a importância das PMEs da construção civil; ii) a promoção e capacitação das PMEs da construção civil na utilização de indicadores; iii) a capacitação de agentes para dar suporte às PMEs através de cursos, estágios, intercâmbios, etc; e iv) o desenvolvimento de competências técnicas no campo de critérios de medição e sistema de indicadores através de convênios com organizações como a FPNQ, Fundação Vanzolini e outras.

Outras soluções que podem ser aplicadas em relação aos problemas citados são: i) a disponibilização de doutores, engenheiros ou técnicos para as PMEs através da concessão de bolsas para que estudem os problemas relatados e apresentem soluções; ii) o desenvolvimento de programas de pesquisas de soluções para os problemas encontrados, subvencionadas através de dotações de recursos; iii) estudar de que maneira as entidades de classe podem ter mais agilidade na prestação de serviços estratégicos na disseminação do conhecimento e da utilização de indicadores, abandonando as práticas desatualizadas e ineficazes que houver; e iv) treinamento de conteúdo teórico e prático para a mão-de-obra operacional.

As organizações devem buscar desenvolver as seguintes competências estratégicas e organizacionais: visão de longo prazo, capacidade de antever tendências, capacidade de gerenciar o risco, etc. (OCDE, 1996). A partir da análise dos problemas apontados, observa-se que as PMEs não certificadas ainda não desenvolvem estas capacidades. Tal observação é corroborada por Muñoz (2001) ao afirmar que o gerenciamento das construtoras é caracterizado por baixa orientação para o futuro.

As PMEs devem compreender que o SGQ é um sistema permanente e de longo prazo, direcionado para a satisfação do cliente, através da melhoria contínua dos produtos e serviços, conforme a visão de Mears (1993). É fundamental que aceitem que a eficiência operacional superior fortalece a posição competitiva das organizações. Quanto à utilização de indicadores, Lantelme (1994) verificou que, em geral, as empresas de construção não possuem sistemas de medição ou quando os têm procuram indicadores mais simples sem ligação com os realmente críticos. No entanto, autores como Ohashi e Melhado (2004) demonstraram a importância da utilização de indicadores em empresas construtoras certificadas. Estes estudos devem ser levados ao conhecimento das PMEs para que se sensibilizem em relação a este aspecto. Como já mencionado, a medição é fundamental para a melhoria contínua.

As razões listadas no relatório McKinsey (1998) e no Estudo Prospectivo da Construção Civil (MDIC, 2003) reforçam a necessidade de que as soluções aqui descritas sejam consideradas pelas PMEs. Estas devem se equipar com técnicas para melhorar sua eficácia técnico-administrativa e seus recursos humanos, estruturando-se de modo a integrar seus sistemas internos e atender às estratégias definidas, conforme preconizado por autores como Nohria e Ghoshal (1997), Dunning (1993) e outros.

\section{Considerações finais}

Procurou-se, através da medição de indicadores, identificar o desempenho das PMEs certificadas e não certificadas. A pesquisa realizada demonstrou, através da comparação de indicadores previamente escolhidos, que as empresas que utilizam critérios de medição apresentam melhores resultados. Salienta-se que os indicadores são 
essenciais para que o nível de desempenho das organizações seja medido.

Observou-se que as PMEs com critérios de medição e sistemas de indicadores estão mais bem preparadas para as mudanças que agitam o ambiente de negócios, além de utilizar melhor os indicadores e ter uma melhor percepção do ambiente externo. Deste modo, estas organizações conseguem rever suas estratégias. Os resultados obtidos na pesquisa comprovam esta afirmação. A utilização de indicadores possibilita às empresas conhecer seus pontos deficientes e traçar estratégias visando o aprimoramento contínuo. Agindo desta forma, podem se adaptar melhor às mutações do ambiente. Com o aprimoramento dos programas existentes, as PMEs obterão melhores condições de sobreviver no ambiente global de nossos dias.
Foi observado, durante as pesquisas realizadas, que a utilização de indicadores de qualidade e produtividade para avaliação de desempenho e melhoria dos processos ainda é incipiente na construção civil. A implantação de sistemas de indicadores é dificultada por fatores característicos deste setor tais como: caráter nômade, produtos únicos e não seriados, longo ciclo de aquisição-usoreaquisição, mão-de-obra de baixa capacitação, dentre outros. Outro obstáculo para sua implantação é a sobrecarga de trabalho e a necessidade de redução de custos, existentes nas atuais condições da construção civil. No entanto, à medida que resultados positivos forem sendo conhecidos, espera-se uma melhor difusão do uso de sistemas de indicadores. Enfim, a utilização de critérios de medição e sistemas de indicadores é um poderoso instrumento de aperfeiçoamento da gestão das empresas.

\title{
Comparing small and medium-sized civil construction enterprises based on performance indicator systems
}

\begin{abstract}
This article evaluates how small and medium-sized enterprises (SMEs) in the civil construction industry that utilize performance indicator systems perform when compared with similar companies that do not use such indicators. SMEs located in the region of Rio de Janeiro were selected, some certified with quality systems and others not. Various indicators (financial, human resources, market and production) were applied to the companies and a comparison was made of the findings. Tests revealed that companies that use performance indicator systems show better results.
\end{abstract}

Keywords: Small and medium-sized enterprises. Civil construction. indicators.

\section{Referências bibliográficas}

ADEMI- Associação de dirigentes de empresas do mercado imobiliário. Pesquisa de acompanhamento e análise do mercado imobiliário: boletins internos. Rio de janeiro: ADEMI, 2005.

ALBAGLI, S. Capacitação, Sensibilização e Informação em Arranjos e Sistemas de MPMENT1. Rio de Janeiro: UFRJ, 2001.

AMORIM, S. R. L. Tecnologia, Organização e Produtividade na Construção. Rio de Janeiro, 1995. Tese - (Doutorado em Engenharia de Produção), Universidade Federal do Rio de Janeiro - UFRJ.

O sistema de indicadores de qualidade e produtividade do clube de Construção do Rio de Janeiro: novas ferramentas para a gerência do canteiro. Florianópolis: VII ETAC, 1998.

BARROS NETO, J. P. Proposta de um modelo de formulação de estratégias de produção para pequenas empresas de construção habitacional. Rio Grande do Sul, 1999. Tese (Doutorado em Administração), Universidade Federal do Rio Grande do Sul.

BETHLEM, A. S. Estratégia empresarial: conceitos, processo e administração estratégica. São Paulo: Atlas, 2004.
CÂMARA BRASILEIRA DA INDÚSTRIA DA CONSTRUÇÃO CIVIL. Disponível em: http://<http://www.cbic.org.br>. Acesso em: 06 set. 2005.

CÂMARA BRASILEIRA DA INDÚSTRIA DA CONSTRUÇÃO CIVIL. Macro-setor da construção produz quase $20 \%$ do PIB (release). São Paulo: Fundação Getúlio Vargas, 2002. Disponível em: http://www.moradia.org.br/BuscaDados.asp. Acesso em: 10 abr. 2005.

COSTA, D. R. Diretrizes para concepção, implementação e uso de sistemas de indicadores de desempenho para empresas de construção civil. Rio Grande do Sul, 2003. Dissertação (Mestrado em Engenharia Civil), Universidade Federal do Rio Grande do Sul.

CROSS, K. F.; LYNCH, R. L. The SMART way to define and sustain success. National Productivity Review. The Journal of Productivity Management, New York, v. 8, n. 1, p. 23-33, 1988.

DUNNING, J. H. Multinational enterprise and the global economy. Workingham: Addison-Wexley, 1993.

FIRJAN - FEDERAÇÃO DAS INDÚSTRIAS DO RIO DE JANEIRO. Cadastro Industrial do Estado do Rio de Janeiro 2003-2004. Rio de Janeiro: FIRJAN, 2003. 
FONSECA, F. L. Metodologia de avaliação de desempenho de empresas construtoras com sistema de gestão de qualidade implementado. Rio de Janeiro, 2006. Dissertação - (Mestrado), PPGEC, Universidade Federal Fluminense.

FORMOSO, C. A.; OLIVEIRA, M.; LANTELME, E. Indicadores de qualidade e produtividade; medidas de desempenho para a construção civil. In: Simpósio da Aplicação da Tecnologia do Concreto, 17, 1994, Campinas. Anais... Campinas, 1994.

FPNQ-FUNDAÇÃONACIONAL PARA OPRÊMIONACIONAL DA QUALIDADE. Caso para estudo: escritório de engenharia Joal Teitelbaum. São Paulo: FPNQ, 2004.

Critérios de excelência: o estado da arte da arte da gestão para a excelência da desempenho. São Paulo, 2002. Disponível em: <http://www.fpnq.org.br> Acesso em: 24 Agosto 2005.

GHALAYINI, A. M.; NOBLE, J. S.; CROWE, T. J. An integrated dynamic performance measurement system for improving manufacturing competitiveness. International Journal of Production Economics, Amsterdam, v. 48, n. 3, p. 207-225, feb.1997.

GRILO, L. et al. Análise da implementação dos princípios de gestão da qualidade em empresas de projeto. Ambiente Construído, Porto Alegre, v. 3, n. 1, p. 55-67, jan./mar., 2003

HRONEC, S. M. Sinais vitais: usando medidas da qualidade, tempo e custo para traçar a rota para o futuro de sua empresa. São Paulo: Makron, 1994.

IBGE - INSTITUTO BRASILEIRO DE GEOGRAFIA E ESTATÍSTICA. Pesquisa Anual da Indústria da Construção 2003. Disponível em <http://www.ibge.gov.br>. Acesso em: 29 Abril 2006.

Pesquisa Estatística 2003. Disponível em: <http://www. ibge.gov.br/estatistica>. Acesso em: 30 Abril 2006.

KAPLAN, R. S.; NORTON, D. R. The balanced scorecardmeasures that drive performance. Harvard Business Review, Boston, v. 70, n. 1, p .71-79, jan./ feb .1992.

A estratégia em ação. Rio de Janeiro: Campus, 1997.

LANTELME, E. M. V. Proposta de um sistema de indicadores de qualidade e produtividade para a construção civil. Porto Alegre, 1994. Dissertação - (Mestrado em engenharia civil), Escola de Engenharia, Universidade Federal do Rio Grande do Sul.

. A utilização de indicadores na avaliação e melhoria do desempenho de processos da construção de edificações: uma abordagem com base em princípios da aprendizagem organizacional. Seminário de doutorado. Curso de Pós-Graduação em Engenharia Civil. Porto Alegre: Escola de Engenharia da Universidade Federal do Rio Grande do Sul, 1999.

LANTELME, E. M. V. ; TZORTZOUPOULOS, P.; FORMOSO, C. T. Indicadores de qualidade e produtividade para a construção civil. Relatório de pesquisa. Porto Alegre: Núcleo Orientado para a Inovação da Edificação, Programa de Pós-Graduação em Engenharia Civil, Universidade Federal do Rio Grande do Sul, 2001.

LITWIN, M. S. How to measure survey reliability and validity. Thousand Oaks: Sage, The Survey Kit, 1995.

LIMA, H. M. R. Concepção e implementação de sistema de indicadores em empresas construtoras de empreendimentos habitacionais de baixa renda. Porto Alegre, 2005. Dissertação - (Mestrado), PPGEC, Universidade Federal do Rio Grande do Sul.

LYNCH, R, L.; CROSS, K. F. Measure up: yardsticks for continuous improvement. Cambridge: Blackwell, 1995.
Promoting Innovation Systems as a Response to the Globalising Learning Economy. Contrato BNDES/FINEP/ FUJB. Rio de Janeiro, 2000.

MCKINSEY GLOBAL INSTITUTE. Produtividade: a chave do desenvolvimento acelerado no Brasil. São Paulo: Mckinsey Brasil, 1998. (relatório).

MEARS, P. How to stop talking about and begin progress toward total quality management. Business horizons, Greenwich, v. 36,1993 , p. 66-68.

MINISTÉRIO DO DESENVOLVIMENTO, INDÚSTRIA E COMÉRCIO EXTERIOR. Disponível em: <http://www. desenvolvimento.gov.br>. Acesso em: 09 Setembro 2005.

MUÑOZ, R. O processo de inovação e difusão tecnológica na construção civil: o caso do plano 100 em Salvador. Salvador, 2001. Dissertação - (Mestrado), Escola de Administração, Universidade de Federal da Bahia.

NEELY, A. et al. Realizing strategy through measurement. International Journal of Operation \& Production Management, Bradford, v. 15, n. 3, p. 140-152, 1992.

Performance measurement system design: should process based approaches be adopted? International Journal Production Economics, Amsterdam, v. 46-47, p. 423-431, 1997.

NOHRIA, N.; GHOSHAL, S. The differentiated networkorganizing multinational corporations for valor creation. San Francisco: Jossey-Bass Publishers, 1997.

OHASHI, E. A. M.; MELHADO, S. B. A importância dos indicadores de desempenho nas empresas construtoras e incorporador as com certificação ISO 9001:2000. In: I Conferência Latino- Americana de Construção Sustentável, X Encontro Nacional do Ambiente Construído, 2004, São Paulo. Anais... São Paulo, 2004.

OLIVEIRA, M.; LANTELME, E. M. V.; FORMOSO, C. T. Sistemas de indicadores de qualidade e produtividade na construção civil: manual de utilização. Porto Alegre: SEBRAE, 1995.

OLIVEIRA, M.; FREITAS, H. Seleção de indicadores para tomada de decisão: a percepção dos principais intervenientes na construção civil. Revista Eletrônica de Administração, Porto Alegre, v. 7, n. 1, mar. 2000. Disponível em: <http://www.read. adm.ufrgs.br/read19/artigo/artigo5.htm>. Acesso em: 17 ago. 2005.

OCDE - ORGANIZAÇÃO PARA A COOPERAÇÃO E DESENVOLVIMENTO ECONÔMICO. The OECD Jobs Strategy - Technology, Productivity and Jobs Creation. Paris, 1996.

PBQP-H. PROGRAMA BRASILEIRO DA QUALIDADE E PRODUTIVIDADE - HABITAÇÃO. Ministério das Cidades. Disponível em: <www.cidades.gov.br/pbqp-h/empresas_ qualificadas.htm > Acesso em: 7 ago. 2005.

- Ministério das Cidades. Disponível em <http://www. cidades.gov.br>. Acesso em: 10 abr. 2006.

QUALIPRO. Sistema de acompanhamento da qualidade e produtividade na construção: manual de coordenação. Rio de Janeiro: Clube da Qualidade na Construção e Universidade Federal Fluminense, 1998.

RIBAULT, J. M.; MARTINET, B.; LEBIDOIS, D. A gestão da tecnologia. Lisboa: D. Quixote, 1995.

RODRIGUEZ, M. V. R. Gestão Empresarial: organizações que aprendem. Rio de Janeiro: Qualitymark, 2002.

SANTANA, P. H. S. Indicadores de Produtividade nas Edificações: um estudo com enfoque na teoria da amostragem. 
Niterói, 1997. Dissertação - (Mestrado em Engenharia Civil), Universidade Federal Fluminense.

SCHIEMANN, W. A.; LINGLE, J. H. Bulls eye: hitting your strategic targets though high impact measurements. New York: The Free Press, 1999.

SENAI - SERVIÇO NACIONAL DE APRENDIZAGEM INDUSTRIAL. 1999. Disponível em < http://www.senai.br > Acesso em: 22 dez. 2004.

SINDUSCON-SP. Setor da Construção em Números. 200. Disponível em: < www.sindusconsp.com.br > Acesso em: 14 nov. 2005.
SINK, D. S.; TUTTLE, T. C. Planejamento e medição para performance. Rio de Janeiro: Qualitymark, 1993.

SOUZA, R.; SAMPAIO, J. C. A.;MEKBEKIAN,G. Sistema de gestão da qualidade para empresas construtoras. São Paulo: Pini, 1995.

SOUZA, D.A. A operacionalização de um sistema de indicadores de qualidade e produtividade na construção civil: um estudo de caso na cidade do Rio de Janeiro. Niterói, 1996. Dissertação - (Mestrado), PPGEC, Universidade Federal Fluminense.

YIN, R. K. Estudo de caso: planejamento e métodos. Porto Alegre: Bookman, 2001.

\section{Sobre os autores}

\section{Luiz Carlos Brasil de Brito Mello}

\section{Sérgio Roberto Leusin de Amorim}

Programa de Pós Graduação em Engenharia Civil, PPGEC, Escola de Engenharia, Universidade Federal Fluminense,

Rua Passo da Pátria 156, sala 365, Bloco D, Niterói, RJ, Brasil, e-mails: luiz.brasil@ gmail.com; sergio.leusin@gmail.com

\section{Renata Albergaria de Mello Bandeira}

Programa de Pós Graduação em Administração - PPGA,

Escola de Administração, Universidade Federal do Rio Grande do Sul,

Rua Washington Luiz, 855, Centro, Porto Alegre, RS, Brasil,

e-mail: re.albergaria@gmail.com 